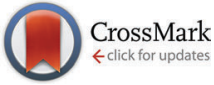

Cite this: Chem. Commun., 2016 52, 2103

Received 15th October 2015, Accepted 4th December 2015

DOI: $10.1039 / c 5 c c 08590 j$

www.rsc.org/chemcomm

\section{Azo ...phenyl stacking: a persistent self-assembly motif guides the assembly of fluorinated cis-azobenzenes into photo-mechanical needle crystals $\dagger$}

\author{
Oleksandr S. Bushuyev, Anna Tomberg, Joanna R. Vinden, Nicolas Moitessier, \\ Christopher J. Barrett* and Tomislav Friščić*
}

\begin{abstract}
We describe a novel, persistent motif of molecular assembly in photomechanical crystals and cocrystals of fluorinated cis-azobenzenes. The azo ...phenyl stacking, preserved upon either chemical substitution or halogen-bonded cocrystallization, guides the assembly of fluorinated cis-azobenzenes into columnar stacks and drives the formation of crystals with needle-like morphologies optimal for photo-mechanical motion.
\end{abstract}

Control over molecular assembly in crystals is central to a range of applications for molecular materials, such as pharmaceuticals, ${ }^{1,2}$ organic semiconductors, ${ }^{3}$ and energy transduction and storage materials. ${ }^{4}$ The latter have recently received much interest, as they include light-harvesting systems which can deform mechanically upon irradiation, and function as artificial muscles, photomechanical robots, and 'molecular machines'. ${ }^{5}$ The understanding and prediction of solid-state molecular assembly, which is key to such designer crystalline materials, has been greatly advanced by an understanding of hydrogen-bonded supramolecular synthons ${ }^{6}$ which, along with a variety of other interactions (e.g. halogen bonds, $\pi-\pi$ interactions), enable the design of crystals of high complexity and functional nature, including pharmaceuticals, explosives, or photo-switchable materials. ${ }^{7-10} \mathrm{~A}$ recent development in the latter context is the discovery that azobenzenes, if obtained as sufficiently thin crystalline plates or needles, readily undergo large-scale light-induced photo-mechanical motion associated with photochemical switching of trans- and cis-geometries. ${ }^{11-13}$ Specifically, crystals of trans-azobenzenes undergo rapid, reversible motion upon exposure to ultraviolet or visible light, ${ }^{11,13}$ while fluorinated cis-azobenzenes, such as cis-1 and cis-2 (Fig. 1a),

Department of Chemistry, McGill University, 801 Sherbrooke St. W. H3A OB8, Montreal, Canada. E-mail: christopher.barrett@mcgill.ca,

tomislav.friscic@mcgill.ca

$\dagger$ Electronic supplementary information (ESI) available: Experimental and characterization data, including summary of X-ray crystallography, calculated densities for selected cis- and trans-azobenzene structures, theoretical QM and intermolecular potential calculations, UV/Vis spectra, mass spectrometry and infrared spectroscopy data. CCDC 1431056-1431064. For ESI and crystallographic data in CIF or other electronic format see DOI: $10.1039 / \mathrm{c} 5 \mathrm{cc} 08590 \mathrm{j}$ undergo light-induced cis $\rightarrow$ trans isomerization accompanied with irreversible bending. ${ }^{14,15}$ Critical to both types of photomechanical motion is the formation of crystals sufficiently long and thin to permit macroscopic bending.

We now report a persistent, previously undescribed azo *..phenyl stacking motif in crystalline fluorinated cis-azobenzenes, leading to columnar 'pringle chip'-like supramolecular architectures ${ }^{16}$ and potentially driving the formation of crystals with thin needle-like morphologies (Fig. 1b and c, Fig. S2, ESI $\dagger$ ) optimal for photo-mechanical motion. For the first time, this molecular assembly motif offers an opportunity to design crystal structures and ensure crystal morphologies ideal for photo-mechanical

a)
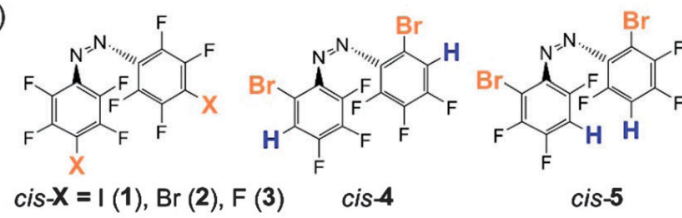

cis-4

cis -5
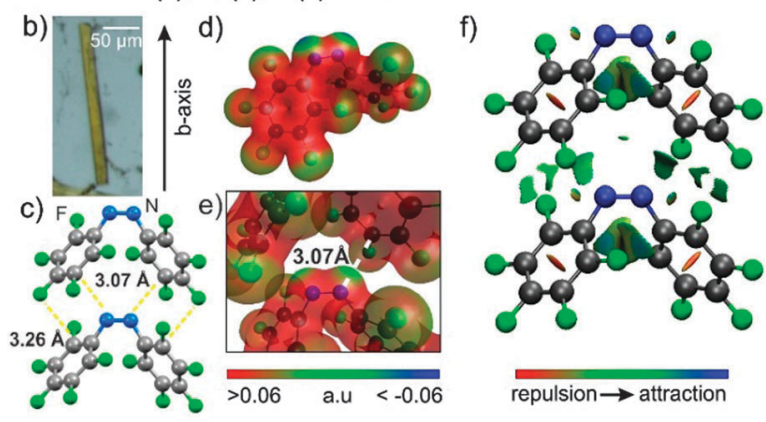

repulsion $\rightarrow$ attraction

Fig. 1 (a) Fluorinated cis-azobenzenes synthesized in this work; (b) typical needle-like crystal morphology observed for fluorinated cis-azobenzenes; (c) stacking of cis-3 parallel to the long axis of crystal needle growth. Molecular electrostatic potential (MEP) calculated for: (d) a single cis-3 molecule and (e) for a pair of cis-3 molecules in an azo...phenyl stack (contours at 0.02 a.u.; red: repulsion; blue: attraction), highlighting the short contact between the azo nitrogen and a meta-positioned $\mathrm{C}-\mathrm{F}$ bond. (f) The $\mathrm{NCl}$ plot for two molecules of cis-3 in an azo... phenyl stack $(\mathrm{NCl}$ contours at 0.5 , electron density threshold 0.2 ), demonstrating notable areas of attraction and repulsion. 
behaviour, advancing applications in light-driven robotics, molecular machines, and artificial muscles. ${ }^{5}$

The azo ..phenyl stacking in fact can be recognized from careful inspection of previously published ${ }^{14 b}$ crystal structures of cis-1 and two polymorphs of cis-2. These consist of onedimensional molecular stacks with repeat distances 5.324(1) (cis-1, CSD code DOCCOP), 5.597(1) A (cis-2, CSD code DOCCEF01) and 5.316(1) $\AA$ (cis-2, CSD code DOCCEF) along the crystallographic $b$-axis, respectively (Fig. S1, ESI $\dagger$ ). Importantly, stacks exhibit unusually short $\mathrm{N}$-. C contacts between azo groups and fluorinated phenyl rings of neighbouring molecules (3.190(5) $\AA$ in cis-1, 3.210(3) $\AA$ and 3.157(3) in polymorphs of cis-2), compared to $\mathrm{N}$...C separation of ca. $3.3 \AA$ expected from van der Waals radii. ${ }^{17}$ Importantly, almost identical stacking is seen in reported ${ }^{14 a}$ cocrystals of cis-1 and cis-2, but not in non-fluorinated cisazobenzenes, ${ }^{18}$ suggesting it may be characteristic for only the fluorinated derivatives. To verify the presence of a persistent stacking motif, we first prepared the fully fluorinated cis-3 (Fig. 1a). $\$$ Crystallization of cis-3 from hexane gave thin needles (Fig. 1b), established by single crystal X-ray diffraction to consist of one-dimensional stacks parallel to the crystallographic $b$-axis, resembling the stacks in cis-1 and -2. In cis-3, stacks are arranged in parallel, characterized by a short $\mathrm{N}$... C separation (3.073(4) A) and a repeat distance of 5.569(3) A.

Formation of identical stacks independent of para-substituent is remarkable and points to a robust self-assembly motif. We undertook a computational analysis of interactions between molecules in stacks of cis-1, cis-2 (for one polymorph only, CSD code DOCCEF01) and cis-3 (see also ESI $\dagger$ ). The molecular electrostatic potential (MEP) analysis revealed that stacking may be supported by two attractive interactions (Fig. 1c-e, Fig. S3 and S4, $\mathrm{ESI} \dagger$ ). One arises between the lone electron pair on each of the azo group nitrogen atoms and meta-carbon atoms in the fluorinated phenyl group of the neighbouring molecule. The other attractive interaction involves the negatively charged rim of the halogen para-substituents and the positively charged carbon atoms in perfluorinated phenyl groups of neighbouring molecules. We also evaluated these noncovalent interactions between two neighbouring molecules by calculating the non-covalent interactions (NCI) index using NCIplot, ${ }^{19}$ which depicts intermolecular interactions by an electron density gradient isosurface. The NCI calculations (Fig. 1f, Fig. S3 and S4, ESI $\dagger$ ) confirm that the above interactions are attractive and may help formation of molecular stacks.

In order to evaluate the importance of azo * phenyl stacking in the crystal packing of cis-3, we calculated intermolecular potentials using the UNI force field, implemented in MercuryCSD (version 3.5.1.). ${ }^{20,21}$ Results reveal that stacking of cis-3 is the single most dominant stabilization factor in the crystal structure, with an intermolecular potential of $-34 \mathrm{~kJ} \mathrm{~mol}^{-1}$ (see ESI, $\dagger$ Fig. S5-S13). The next most negative intermolecular potential in crystal structure of cis-3 was at $-16 \mathrm{~kJ} \mathrm{~mol}^{-1}$. Similar results were obtained for the published structure of cis-1 (CSD code DOCCOP), where molecular stacks provide a stabilization of $-54 \mathrm{~kJ} \mathrm{~mol}^{-1}$, the next lowest intermolecular potential being $-25 \mathrm{~kJ} \mathrm{~mol}^{-1}$. For both known cis-2 polymorphs (CSD codes DOCCEF and DOCCEF01), azo $\cdots$ phenyl stacks provide a stabilization of $-44 \mathrm{~kJ} \mathrm{~mol}^{-1}$ and $-50 \mathrm{~kJ} \mathrm{~mol}^{-1}$, with the next most negative intermolecular potential at $-22 \mathrm{~kJ} \mathrm{~mol}^{-1}$ (see ESI, $\dagger$ Fig. S5-S13). These calculations point to azo $\cdots$ phenyl stacking as the most stabilizing interaction in crystalline fluorinated cis-azobenzenes.

To explore the robustness of azo $\cdots$ phenyl stacking to modification of fluorinated cis-azobenzenes, we synthesized and grew single crystals of $c i s-\mathbf{4}$ and $c i s-5$, in which the fluorinated phenyl rings bear a bromine atom in 2-position, and a hydrogen atom in positions 3 and 5, respectively (Fig. 1a). Both compounds form thin needle-shape crystals and crystal structure determination revealed antiparallel azo * phenyl stacks along the crystallographic $b$-direction, i.e. coincident with the crystal needle axis (Fig. 2). In cis-4 the molecules are arranged with a repeat distance of 5.661(3) within each stack. In cis-5 the repeat distance is 5.608(4) $\AA$, with nearest-neighbour molecules in each stack mutually rotated by $90^{\circ} . \S$

All herein explored fluorinated cis-azobenzenes, as well as previously reported $c i s-\mathbf{1}$, cis-2 and their cocrystals, ${ }^{14}$ persistently form thin needle crystals from different solvents $\left(\mathrm{CH}_{2} \mathrm{Cl}_{2}\right.$, acetonitrile, hexanes, ethyl acetate), with the needle axis always parallel to direction of molecular stacks, suggesting that azo...phenyl stacking can govern macroscopic crystal shape and, therefore, potential photo-mechanical behaviour.

Photo-mechanical properties were explored for needles of cis-3 which underwent bending under irradiation with visible (488 nm) laser light. In contrast to crystals of cis-1 and cis-2, that bend away from the light source, needles of cis-3 initially bend away and, after a few seconds of continuous irradiation, reverse the direction of motion and instead bend towards the light source (Fig. 3a-d). The maximum observed crystal tip deflection was $c a .5^{\circ}$. Similar to cis-1 and cis-2, irradiation of cis-3 leads to cis-trans isomerization, confirmed through reaction monitoring by powder X-ray diffraction (PXRD, Fig. 3e). Irradiation of bulk powder leads to disappearance of X-ray reflections of cis-3 and the appearance of new reflections, largely matching those for trans-3 which was also separately prepared and characterized by X-ray single crystal diffraction.

A tentative explanation for the unusual bending behaviour of cis-3 compared to cis-1 and cis-2 may be in the relative densities of corresponding cis- and trans-compounds. Whereas the structures of trans-1 and $\mathbf{- 2}$ exhibit densities only $\sim 3 \%$ higher than those of cis-isomers, the density of trans-3 is $>5 \%$ higher
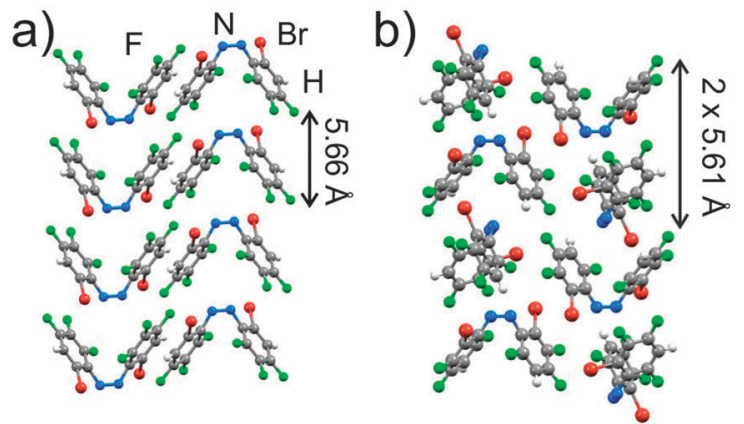

Fig. 2 Azo...phenyl molecular stacking in single crystals of: (a) cis-4 and (b) cis-5. 

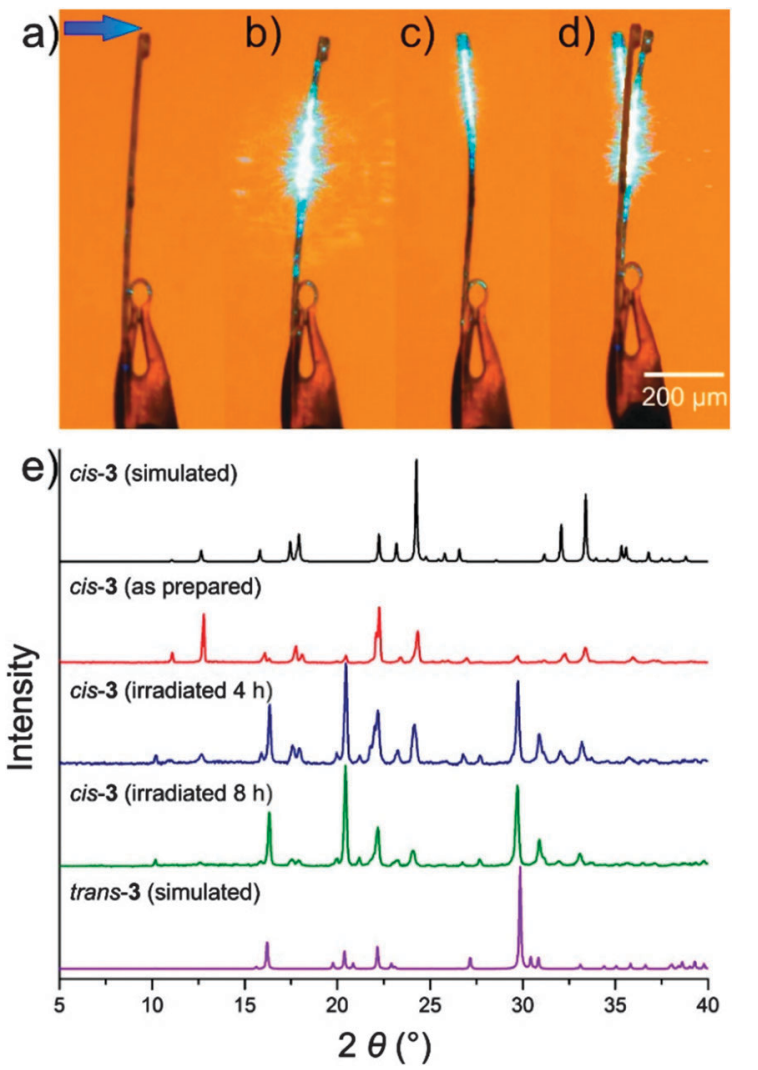

Fig. 3 Bending of a single crystal of cis -3 upon irradiation with a $5 \mathrm{~mW}$, $488 \mathrm{~nm}$ laser (arrow indicates direction of irradiation): (a) before irradiation; (b) after 10 seconds of irradiation; (c) after 30 seconds of irradiation; (d) image superimposing three snapshots of crystal motion. (e) PXRD patterns for bulk cis-3 at different irradiation times, demonstrating gradual conversion to trans-3.

than for cis-3 (Table S3, also see ESI†). Consequently, it may be that during photochemical transformation the density of irradiated material is first reduced due to amorphisation, resulting in photo-expansion and bending away from the light source, as indeed observed for irradiated single crystals and cocrystals of cis-1 and $-2 .{ }^{14}$ Subsequent crystallization of the more dense trans-3 could steer crystal motion in the opposite direction, towards the light. Importantly, cis-5 with a trans-isomer of notably higher density $(\sim 8 \%)$ than its $c i s$-form demonstrates a similar photoresponse, while cis-4 with $\sim 3 \%$ denser trans-isomer exhibited a much weaker response to irradiation (Table S3, ESI $\dagger$ ) $\ddagger$ Whereas the Kobatake group recently demonstrated how varying the radiation wavelength can alter the direction of photo-mechanical motion for diarylethylene crystals, ${ }^{22}$ in our case the course of bending did not change upon irradiation at $405 \mathrm{~nm}, 488 \mathrm{~nm}$ and $532 \mathrm{~nm}$.

The stabilizing interaction of the fluorinated phenyl group with negatively charged rim of the iodine or bromine substituents (S3 and S4, ESI $\dagger$ ) leaves the azo *..phenyl stacks in cis-1 and cis-2 decorated with positive $\sigma$-holes potentially accessible for halogen bonding. Consequently, we tested if azo *..phenyl stacking in cis-1 is retained upon halogen-bonded cocrystallization ${ }^{23,24}$ with 1,4diazabicyclo[2.2.2] octane (dabco), 1,4-dithiane and 4-vinylpyridine a)

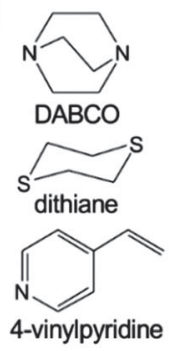

c)

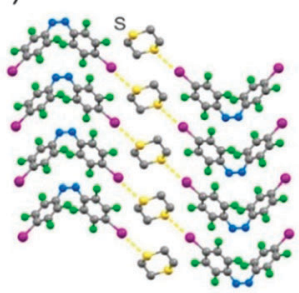

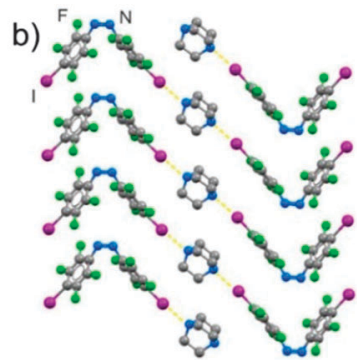

d)

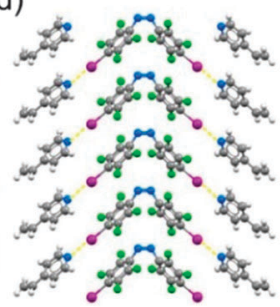

Fig. 4 (a) Herein explored halogen bond acceptors. Fragments of crystal structures of cocrystals of cis-1 with: (b) dabco; (c) dithiane and (d) 4-vinylpyridine, demonstrating halogen bond formation lateral to azo ...phenyl stacking of cis-1. Molecular stacks are parallel to the crystallographic $b$-axis, i.e. the long axis of the needle-shaped crystal.

acceptors (Fig. 4a). Cocrystallization of cis-1 with dabco from dichloromethane $\left(\mathrm{CH}_{2} \mathrm{Cl}_{2}\right)$ gave needle-like crystals of (cis-1)(dabco), consisting of antiparallel azo *.phenyl stacks of cis-1 (repeat distance: $6.331(1) \AA ̊$ ) , laterally decorated by $\mathrm{I} \cdots \mathrm{N}$ halogen bonds to dabco molecules $(\mathrm{I} \cdots \mathrm{N}$ distance: $2.758(2) \AA)$ that bridge neighbouring stacks. Cocrystallization of cis-1 and dithiane from $\mathrm{CH}_{2} \mathrm{Cl}_{2}$ gave long needles of (cis-1)(dithiane), consisting of antiparallel stacks of cis-1 (repeat distance: 5.4079(4) $\AA$ ) bridged by dithiane molecules connected via $\mathrm{I} \cdots \mathrm{S}$ halogen

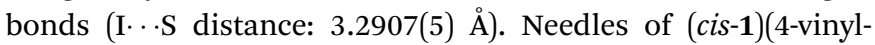
pyridine $)_{2}$ were obtained from a $\mathrm{CH}_{2} \mathrm{Cl}_{2} / 4$-vinylpyridine mixture $(90: 10, \mathrm{v} / \mathrm{v})$ by slow evaporation. Single crystal X-ray diffraction revealed antiparallel stacks of cis-1 (stack repeat: 5.4157(7) ̊̊) laterally decorated by halogen-bonded 4-vinylpyridine acceptors $(\mathrm{I} \cdots \mathrm{N}$ distance: $2.780(2) \AA)$. Synthesis and structures of $($ cis-1)(dabco), (cis-1)(dithiane) and (cis-1)(4-vinylpyridine) $)_{2}$ demonstrate azo * phenyl stacking of fluorinated cis-azobenzenes as a persistent motif for crystal engineering: azo $\cdots$ phenyl stacks were in all cases aligned with the long needle axis of the crystal and evaluation of intermolecular potentials revealed azo * phenyl stacks as the most significant stabilizing motifs in all three cocrystals (Fig. S5-S13, ESI $\dagger$ ).

In conclusion, we identified a new persistent supramolecular stacking motif based on fluorinated cis-azobenzene units. The azo *..phenyl stacking has been observed for all herein described compounds, as well as all previously reported structures of fluorinated cis-azobenzene crystals and cocrystals. Stacking persists upon varying the type and position of substituents $(\mathrm{H}, \mathrm{F}$, $\mathrm{Br}, \mathrm{I})$ on the aryl rings, and upon halogen-bonded cocrystallization, which permitted the organisation of other molecules in onedimensional fashion. ${ }^{24}$ All fluorinated cis-azobenzene crystals and cocrystals explored herein and in previous work ${ }^{14}$ appear as elongated needles, with azo ‥phenyl molecular stacks parallel 
to the crystal long axis. We therefore believe that azo $\cdots$ phenyl stacking of fluorinated cis-azobenzenes might allow both the predictable organization of these photo-mechanically active molecules into columnar 'pringle'-like supramolecular architectures, as well as encourage the growth of resulting crystals into needles, the ideal morphology for photo-mechanical effects and devices. Also described here is the unique observation of two distinct and opposite photo-mechanical regimes in the same crystal, resulting in bending either towards or away from visible light, depending on the time-scale of irradiation. We believe that azo $\cdots$ phenyl stacking of fluorinated cis-azobenzenes may be developed into a rational tool to simultaneously control the crystal structure and permit ideal crystal morphologies for various applications. ${ }^{24}$ In that context, we are now investigating photo-mechanical behaviour of the herein reported cocrystals.

We thank Profs X. Ottenwaelder and D. S. Bohle for access to X-ray diffraction facilities. O.S.B. acknowledges a Vanier Canada Graduate Fellowship and a Ludo Frevel Crystallography Scholarship. C. B. and T. F. thank the NSERC Discovery Grant program and the Canada Foundation for Innovation for support.

\section{Notes and references}

‡ As cis-o-fluoro-azobenzenes exhibit long thermal half-lives, all herein prepared azobenzenes were nearly quantitatively converted into the cis-form by $532 \mathrm{~nm}$ laser irradiation and then crystallized in the dark. ${ }^{25}$ trans-isomers were also prepared and structurally characterized by single crystal X-ray diffraction. Crystallographic data for all new structures is summarised in the ESI $\dagger$ and submitted in the CSD (CCDC codes 1431056-1431064).

$\S$ Change in orientation of molecules in cis-5 azo $\cdots$ phenyl stacks may be the result of $\mathrm{C}-\mathrm{H} \cdots \mathrm{N}$ contacts in the stacks (Fig. S14, ESI $\dagger$ ).

1 (a) N. Shan and M. J. Zaworotko, Drug Discovery Today, 2008, 13, 440; (b) A. Nangia, Acc. Chem. Res., 2008, 41, 595; (c) D. V. Soldatov, J. Chem. Crystallogr., 2006, 36, 747.

2 (a) D.-K. Bučar, J. A. Elliott, M. D. Eddleston, J. K. Cockcroft and W. Jones, Angew. Chem., Int. Ed., 2015, 54, 249; (b) E. R. Tiekink, Chem. Commun., 2014, 50, 11079.

3 (a) H. T. Black and D. F. Perepichka, Angew. Chem., Int. Ed., 2014, 53, 2138; (b) J. E. Anthony, Nat. Mater., 2014, 13, 773; (c) A. N. Sokolov, T. Friščić and L. R. MacGillivray, J. Am. Chem. Soc., 2006, 128, 2806.

4 (a) M. Morimoto and M. Irie, J. Am. Chem. Soc., 2010, 132, 14172; (b) I. A. Khan, A. Badshah, A. A. Altaf, N. Tahir, N. Haider and M. A. Nadeem, RSC Adv., 2015, 5, 9110.

5 (a) J. M. Abendroth, O. S. Bushuyev, P. S. Weiss and C. J. Barrett, ACS Nano, 2015, 9, 7746; (b) M.-M. Russew and S. Hecht, Adv. Mater., 2010, 22, 3348; (c) M. Irie, T. Fukaminato, K. Matsuda and S. Kobatake, Chem. Rev., 2014, 114, 12174.

6 (a) G. R. Desiraju, J. Am. Chem. Soc., 2013, 135, 9952; (b) G. R. Desiraju, Angew. Chem., Int. Ed., 1995, 34, 2311.

7 (a) P. Metrangolo, F. Meyer, T. Pilati, G. Resnati and P. Metrangolo, Acc. Chem. Res., 2008, 47, 6114; (b) A. Mukherjee, S. Tothadi and G. R. Desiraju, Acc. Chem. Res., 2014, 47, 2514; (c) C. B. Aakeröy,
M. Baldrighi, J. Desper, P. Metrangolo and G. Resnati, Chem. - Eur. J., 2013, 19, 16240.

8 (a) A. Sun, J. W. Lauher and N. S. Goroff, Science, 2006, 312, 1030; (b) G. W. Coates, A. R. Dunn, L. M. Henling, J. W. Ziller, E. B. Lobkovsky and R. H. Grubbs, J. Am. Chem. Soc., 1993, 120, 3641; (c) T. Hoang, J. W. Lauher and F. W. Fowler, J. Am. Chem. Soc., 2002, 124, 10656.

9 (a) Ö. Almarsson and M. J. Zaworotko, Chem. Commun., 2004, 1889; (b) J. W. Steed, Trends Pharmacol. Sci., 2013, 34, 185; (c) O. Bolton and A. Matzger, Angew. Chem., Int. Ed., 2011, 50, 8960.

10 (a) S. Ghorai, J. C. Sumrak, K. M. Hutchins, D.-K. Bučar, A. V. Tivanski and L. R. MacGillivray, Chem. Sci., 2013, 4, 4304; (b) K. A. Wheeler, S. H. Malehorn and A. E. Egan, Chem. Commun., 2012, 48, 519; (c) S. Kobatake, S. Takami, H. Muto, T. Ishikawa and M. Irie, Nature, 2007, 446, 778.

11 O. S. Bushuyev, T. Singleton and C. J. Barrett, Adv. Mater., 2013, 25, 1796.

12 (a) N. K. Nath, L. Pejov, S. M. Nichols, C. Hu, N. Saleh, B. Kahr and P. Naumov, J. Am. Chem. Soc., 2014, 136, 2757; (b) N. K. Nath, M. K. Panda, S. C. Sahoo and P. Naumov, CrystEngComm, 2014, 16, 1850.

13 H. Koshima, N. Ojima and H. Uchimoto, J. Am. Chem. Soc., 2009, 131, 6890.

14 (a) O. S. Bushuyev, T. C. Corkery, C. J. Barrett and T. Friščić, Chem. Sci., 2014, 5, 3158; (b) O. S. Bushuyev, A. Tomberg, T. Friščić and C. J. Barrett, J. Am. Chem. Soc., 2013, 135, 12556.

15 For studies of crystal bending, see: (a) S. Ghosh, M. K. Mishra, S. B. Kadambi, U. Ramamurty and G. R. Desiraju, Angew. Chem., Int. Ed., 2015, 54, 2674; (b) C. M. Reddy, R. C. Gundakaram, S. Basavoju, M. T. Kirchner, K. A. Padmanabhan and G. R. Desiraju, Chem. Commun., 2005, 3945; (c) M. K. Panda, S. Ghosh, N. Yasuda, T. Moriwaki, G. D. Mukherjee, C. M. Reddi and P. Naumov, Nat. Chem., 2015, 7, 65.

16 For other solid-state photoreactive stacking designs, see: (a) J. W. Lauher, F. W. Fowler and N. S. Goroff, Acc. Chem. Res., 2008, 41, 1215; (b) A. N. Sokolov, T. Friščić, S. Blais, J. A. Ripmeester and L. R. MacGillivray, Cryst. Growth Des., 2006, 6, 2427.

17 A. Bondi, J. Phys. Chem., 1964, 68, 441.

18 (a) G. C. Hampson and J. M. Robertson, J. Chem. Soc., 1941, 409; (b) N. J. Bunce, G. Ferguson, C. L. Forber and G. J. Stachnyk, J. Org. Chem., 1987, 52, 394; (c) I. Núñez, E. Merino, M. Lecea, S. Pieraccini, G. P. Spada, C. Rosini, G. Mazzeo, M. Ribagorda and M. C. Carreño, Chem. - Eur. J., 2013, 19, 3397.

19 (a) E. R. Johnson, S. Keinan, P. Mori-Sánchez, J. Contreras-García, A. J. Cohen and W. Yang, J. Am. Chem. Soc., 2010, 132, 6498; (b) A. Otero de la Roza, E. R. Johnson and J. Contreras-García, Phys. Chem. Chem. Phys., 2012, 14, 12165.

20 (a) A. Gavezzotti, Acc. Chem. Res., 1994, 27, 309; (b) A. Gavezzotti and G. Filippini, J. Phys. Chem., 1994, 98, 4831.

21 C. C. P. da Silva, R. de O. Pepino, C. C. de Melo, J. C. Tenorio and J. Ellena, Cryst. Growth Des., 2014, 14, 4383.

22 D. Kitagawa, R. Tanaka and S. Kobatake, Phys. Chem. Chem. Phys., $2015,17,27300$

23 For azobenzene cocrystals, see: (a) D. Fox, P. Metrangolo, D. Pasini, T. Pilati, G. resnati and G. Terraneo, CrystEngComm, 2008, 10, 1132; (b) C. B. Aakeröy, S. Panikkattu, P. D. Chopade and J. Desper, CrystEngComm, 2013, 15, 3125; (c) M. Saccone, G. Terraneo, T. Pilati, G. Cavallo, A. Priimagi, P. Metrangolo and G. Resnati, Acta Crystallogr., Sect. B: Struct. Sci., Cryst. Eng. Mater., 2014, 70, 149; (d) A. Priimagi, G. Cavallo, P. Metrangolo and G. Resnati, Acc. Chem. Res., 2013, 46, 2686.

24 J. W. Lauher, F. F. Fowler and N. S. Gorrof, Acc. Chem. Res., 2008, 41, 1215.

25 (a) D. Bléger, J. Schwarz, A. M. Brouwer and S. Hecht, J. Am. Chem. Soc., 2012, 134, 20597; (b) A. A. Beharry, O. Sadovski and G. A. Woolley, J. Am. Chem. Soc., 2011, 133, 19684. 